\title{
Analysis of mechanical automation control forging and visualization
}

\author{
Caifang Zhang
}

\author{
Chongqing Vocational Institute of Engineering, Chongqing, 400037, China
}

\begin{abstract}
Keywords: automatic control; forging; visualization;
\end{abstract}
\begin{abstract}
. the mechanical production process, the production process requirements are very strict, and the production technology has a high demand. Automated production machinery, largely simplifies the original mode of production, and the production efficiency is greatly improved. The forging process of the machinery, and automation technology are integrated effectively, so as to realize the automatic forging machine. However, for the forging technology of automation, there are still shortcomings, or gap distance intelligent. Therefore, through the control of visual operation, can be based on automation production, efficient production of the further implementation of machinery.
\end{abstract}

\section{Introduction}

Industry develops rapidly and mechanical industry is called a leading industry among all industries. For production and manufacturing of machinery, manufacturing process directly affects final quality of mechanical products. When technologies innovate and develop constantly nowadays, automatic production technology of machinery is applied to mechanical forging widely. Automatic forging technology can not only reduce use of workers but also achieve good effect in saving cost and improving product quality. Although automatic forging technology of machinery has been widely used, its technical shortages still exist. Since automation technology cannot reach the stage of intelligent control, manual control is still needed in most cases. Thus, visual control system obtains development and application, and it is combined with automation and control technology to realize the control production technology that can be monitored.

\section{Analysis of mechanical forging}

Mechanical industry and manufacturing industry are basic ones of Chinese industry. For mechanical production, forging technology is quite important. Standard of forging technology decides product quality and its technical core lies on how to product high-quality mechanical products by production control of workshops, management of production equipment and control of product quality.

\subsection{Analysis of features of mechanical products}

In industrial production, the attention paid to mechanical production is high. The reason for this is that mechanical production has high requirements for quality of mechanical products and quality of machinery directly affects safety of its use field. Therefore, there are several basic requirements for mechanical products, as shown in the following.

Firstly, forging processing is needed by mechanical products. There are some differences between forging and manufacturing. Usually, forging need implement high-temperature processing for raw materials of products and high purity is needed. Only in this way can final quality of mechanical products be ensured. In the process of forging, it is necessary to process machinery, such as polishing and shaping, to realize quality assessment criterion of products.

Secondly, careful consideration and examination are needed in the process in which mechanical products are used. Since products' use environment is complicated, mechanical products must have some features like corrosion resistance, high temperature resistance and high pressure resistance etc. Use environment of mechanical products is complicated and conditions of their use are rigorous, which causes the need to check mechanical products strictly. In the process of production, forging technology can satisfy the foregoing requirements well and thus qualified mechanical products can be produced. 
Thirdly, regular repair and maintenance are needed for mechanical products. Existence of repair and maintenance imply machinery needs have the feature that it is repairable to some extent. Generally, mechanical products mainly use metal as their raw materials. Forged metal

\subsection{Technical analysis of mechanical forging}

Mechanical forging technology has some relationship with mechanical manufacturing technology, but there are still many significant differences between the two. Generally, forging technology need process mechanical raw materials repeatedly and raw materials of mechanical production are metal under general conditions. Since metal has re-moldability, test on load bearing and abrasion resistance of metal can be enlarged by forging processing in the process of molding and thus requirements of high-quality mechanical products can be realized.

Forging equipment and forging technical supplement each other, and man forging devices have shaping and separating process. Mechanical products belong to metal forming products and equipment of forging mechanical devices is metal equipment as well. Thus, in the process of forging, it is essential to analyze forged metal to ensure there will be no accidents.

\section{Analysis of mechanical automation forging}

Industrial production has developed rapidly in recent years and a number of high and new technologies are applied to industrial production. For mechanical production, high and new technologies are widely applied to the process of mechanical production since machinery holds a leading position in many industries. In detail, application of automation technology brings huge impacts on mechanical production industry. Thus, effective improvement in cost saving and product quality can be achievement.

\subsection{Mechanical automation technology}

Mechanical manufacture and automation is a hot industry at present. Main production areas of machinery manufacturing are workshops. Factory workers are main members of machinery workshops. To ensure efficiency of machinery production, factory workers usually need work overtime to finish a great deal of work related to machinery production. Especially production of machine parts need be supervised, managed and produced by a number of workers. As the latest technology is applied profoundly and constantly, automation technology begins to be applied to machinery production and can implement engineering management effectively and ensure quality of machinery production. In the process of machinery production, automation technology is mainly applied to control. Generally, machinery production belongs to flow line production and automation technology can realize automatic flow line production. Therefore, use of workers is reduced largely. For advantages of automation technology, they may be elaborated in the following aspects.

Firstly, automation technology reduces use of factory workers, so production cost is reduced. Automation technology can realize flow line automatic production and implement previous work of factory workers to some extent. With respect to use of workers, it realizes simplification. Therefore, expenditures caused by use of workers are reduced. Accordingly, production cost is saved and production quality is improved.

Secondly, automation production technology can reduce errors appearing in production effectively. In the process of machinery production, a number of data and specifications are needed. In previous production of workshop, factory workers carried out control of quality. Errors and impacts of subjective factors would appear easily in the process in which they controlled specifications and standards. Thus, efficiency of production could not be improved and quality of production cannot be ensured to some extent. All of these have impacts on production lines. Therefore, application of automation technology can set all parameters and requirements of production in advance so that automatic control of production can be realized and impacts of errors and subjective factors in production can be reduced as well.

\subsection{Analysis about forging technology of mechanical automation}

For forging technology of machinery, post-combination processing via automation technology can improve efficiency of machinery production and product quality. In forging technology, several operations, for instance, high-temperature iron smelting, are needed. For operation of factory 
workers, they cannot control time accurately but affect product quality to some extent. Thus, in the process of mechanical forging, automation technology is introduced. Automation technology can implement specific operation at specific sites and time, so forging work of machinery can be finished accurately. Besides, automatic forging technology can realize machinery forging with high quality and high efficiency. For instance, the job that raw materials are added repeatedly is needed in welding operation of forging. There are strict requirements for angles, time difference and amount of addition. If addition is finished by workers, there will be two disadvantages. Firstly, it is impossible to ensure time different and amount of addition. Secondly, workers' operation cannot be performed continuously. However, application of automation technology can realize accurate operation and realize high-efficient production.

\section{Analysis about visual control of mechanical forging}

In mechanical forging technology, automation technology is introduced, which can improve quality of forged products to a large extent. However, automation technology has failed to realize real intelligent control yet. For many operations, constructors are still needed to make adjustment. Nevertheless, in production activities, simple automation control technology still has shortages since production environment has impacts on safety of factory workers.

As video technique develops continuously, visual control technology is applied. In the process of mechanical forging, visual control technique can be introduced similarly. In the process of mechanical forging, visual technique usually need satisfy the following conditions. Only in doing so can it exert its role in the process of forging to the largest extent.

Firstly, visual technique need have high-definition video collecting devices. When forging mechanical equipment works, install detecting cameras at specific locations. Thus, each link of production can be learned clearly in the process of forging. In addition, devices collecting video images can be installed in workshops. At such monitoring and collecting devices, engineers can carry out remote supervision to ensure transparency and visualization of each production link.

Secondly, detect production links according to visual system and ensure there is intelligent operating system simultaneously. Production people may analyze collected data in accordance with demands of production and thus implement manual control operation. In control and automatic control field, real intelligent control system has not been realized up to now. Therefore, in order to realize production of mechanical products with high efficiency and high quality, it is necessary to combine and process the two control systems. In most cases, automatic control system can finish some problems in mechanical forging. Nevertheless, in the shaping process of machinery, we need focus on mastering details of mechanical products and thus final products can be perfected. Consequently, quality of mechanical products can be improved further via visual control system.

\section{Conclusion}

Quality requirements of mechanical products are high and such products need accept long-term tests in use. In the process in which mechanical products are produced, many high and new technologies are applied to mechanical production. Automation control technology solves the problem that lots of manpower is taken largely and can improve production quality of mechanical products as well as production efficiency. However, since automation production of machinery has not realized intelligent production, automatic production of machinery still needs people's control. Visual control system technology is just proposed according to the technical difficult point. Visual system monitors and analyzes key production links via detection equipment and production people implements real-time detection. Thus, remote operation can be carried out for forging equipment. Thus, intelligent control of mechanical forging is ensured. In all, there is much development in both application of automation technology and visual control to enhance mechanical forging technology. 


\section{References}

[1] Song Chao. Discussion on Mechanical Automation Technology and Its Application to Mechanical Manufacture [J]. Huadong Technology: Academic Edition, 2013(1):38-40.

[2] Hu Kuohai. Discussion on Mechanical Automation Technology and Its Application to Mechanical Manufacture [J]. Mechanical and Electrical Information, 2012(30): 115-116.

[3] Jiang Wei and Zhang Zuoqiang. Use and Configuration of Hydraulic Machinery Automation Elements at Sanshenggong Hydropower Station (Phase II). Private Science and Technology, 2012(10):70-71.

[4] Bi Tianxiang, Liu Gang, Zhu Gengwu and Yu Yuhong. Application of Deep Hole Working Technique to Cement Mechanical Manufacture [J]. Cement Technology, 2013(1):81-84.

[5] Yuan Xiaochu. Modes of Establishing Intellectual Property Organizations in Mechanical Manufacturing Enterprises [J]. Journal of Harbin Institute of Vocational Technology, 2007(4):203-208. 